\title{
Alteration of miRNA profiles by ionizing radiation in A549 human non-small cell lung cancer cells
}

\author{
SANGSU SHIN ${ }^{1}$, HWA JUN CHA ${ }^{1}$, EUN-MEE LEE ${ }^{1}$, SU-JAE LEE ${ }^{2}$, SUNG-KEUM SEO ${ }^{3}$, \\ HYEON-OK JIN ${ }^{3}$, IN-CHUL PARK ${ }^{3}$, YOUNG-WOO JIN ${ }^{4}$ and SUNGKWAN AN ${ }^{1,5}$ \\ ${ }^{1}$ Functional Genoproteome Research Centre, Konkuk University, Seoul 143-701; ${ }^{2}$ Department of Chemistry, \\ Hanyang University, Seoul 133-791; ${ }^{3}$ Laboratory of Functional Genomics, Korea Institute of Radiological \\ and Medical Sciences, Seoul 139-706; ${ }^{4}$ Division of Radiation Effect Research, Radiation Health \\ Research Institute of KHNP, Seoul 132-703; ${ }^{5}$ LIFEnGENE Inc., Seoul 143-701, Korea
}

Received December 22, 2008; Accepted March 26, 2009

DOI: 10.3892/ijo_00000315

\begin{abstract}
Ionizing radiation (IR) is widely used in cancer treatment and in biological studies. It disrupts cellular homeostasis through multiple mechanisms including changes of the expression profile of genes. Although microRNAs (miRNAs) have recently been recognized as important posttranscriptional regulators and are involved in various biological processes, whether miRNAs play any roles in the cellular response to IR, is not well examined. We investigated the profile of miRNA expression following IR in the human lung carcinoma cell line A549, and the expression profiles of IR-responsive miRNAs were confirmed by qRT-PCR. The target mRNAs of IR-responsive miRNAs were predicted with a target prediction tool. Microarray analysis identified 12 and 18 miRNAs in 20- and 40 Gy-exposed A549 cells, respectively, that exhibited more than 2 -fold changes in their expression levels. Of these, four were changed in only 20Gy-treated cells, ten only in 40-Gy-treated cells, and eight miRNAs were found to change after both treatments. qRTPCR analysis of a subset of the miRNAs showed patterns of regulation as the microarray data, although the magnitude of the changes differed in the two data sets. Target prediction for IR-responsive miRNAs suggests that they target genes related to apoptosis, regulation of cell cycle, and DNA damage and repair. Taken together, these data suggest that miRNA expression is affected by radiation, and they may be involved in the regulation of radiation responses.
\end{abstract}

\section{Introduction}

MicroRNAs (miRNAs), non-coding RNAs of about 22 nucleotides in length, are critical regulators of the translation of specific target mRNAs (1-4). miRNAs usually direct the

Correspondence to: Dr Sungkwan An, Functional Genoproteome Research Centre, Konkuk University, 1 Hwayang-dong, Gwangjin-gu, Seoul 143-701, Korea

E-mail: ansfgrc@konkuk.ac.kr

Key words: microRNA, ionizing radiation, human non-small cell lung cancer cell, A549
miRNA-induced silencing complex to target mRNAs through partial sequence complementarity to the 3' UTRs of target miRNAs, resulting in the suppression of their translation (1,5-7). However, alternative functions of miRNAs, such as the activation of gene expression through interactions with complementary sequences in a gene promoter or the 5' UTR of mRNAs $(8,9)$, have also begun to emerge. The primary miRNA is initially transcribed by RNA polymerase II and processed to a stem-loop-containing precursor miRNA (pre-miRNA) by the microprocessor complex in the nucleus (10-12). Finally, the pre-miRNA is exported to the cytoplasm where it is cleaved by Dicer to form the mature miRNA (5,13-15).

As regulators of mRNA translation, miRNAs are involved in many biological processes including developmental timing, stem cell function, cellular differentiation, proliferation, apoptosis, cell cycle, and diseases such as cancer (16-20). It is known that a single miRNA can target multiple genes and a given gene may also be targeted by multiple miRNAs (21-24). Interestingly, miRNAs may be useful as biomarkers for the identification classification and tissue origin identification of various tumors (25).

Ionizing radiation (IR) is broadly used in cancer treatment and in biological studies. It induces oxidative molecules that cause damage to DNA, proteins, and lipids in the cells (26). The cellular radiation response, which may include DNA repair, cell cycle arrest, and apoptosis, is regulated by numerous genes, such as ataxia-telangiectasia mutated (ATM), tumor suppressor protein p53 (TP53) and all its downstream targets, and DNA damage checkpoint-related genes including cyclindependent kinases (CDKs) and CDC25 family proteins (27-30). The radiation sensitivity of a cell is affected by many factors including cell growth rate, DNA ploidy, and the presence of inhibitors of DNA repair or histone deacetylation (31-34). Furthermore, the activity of genes such as Akt/protein kinase B (PKB) influences the radiosensitivity of non-small cell lung cancer cell lines $(35,36)$.

This study was designed to test the hypothesis that miRNA are involved in the cellular responses to IR. To test this hypothesis, we first conducted a miRNA microarray experiment with which we identified radiation-responsive miRNAs. We 
then confirmed the expression pattern of a subset of these miRNAs by qRT-PCR. Our data indicate that several miRNAs exhibit radiation-responsive changes in expression, suggesting that miRNAs are involved in the cellular responses to IR.

\section{Materials and methods}

Cell culture. The human lung carcinoma cell line A549 was cultured at $37^{\circ} \mathrm{C}$ in RPMI-1640 containing $10 \%$ fetal bovine serum (FBS) and antibiotics at in a humidified chamber supplemented with $5 \% \mathrm{CO}_{2}$. At one day before irradiating, $5 \times 10^{5}$ cells were seeded on $60-\mathrm{mm}$ culture dishes.

Irradiation and RNA preparation. Following $24 \mathrm{~h}$ in culture, the cells were exposed to either 20 or 40 Gy of gamma IR with a Gammacell ${ }^{\circledR} 3000$ Elan irradiator $\left({ }^{137} \mathrm{Cs} \gamma\right.$-ray source; MDS Nordin, ON, Canada). The irradiated cells were then maintained for $24 \mathrm{~h}$ before extracting total RNA with TRIzol ${ }^{\mathrm{R}}$ reagent (Invitrogen, CA, USA) according to the manufacturer's protocol. For the microarray studies, the quality and concentration of RNA samples were determined with an Agilent 2100 Bioanalyzer (Agilent Technologies, CA, USA) and an Ultrospec 3300 Pro UV/Visible Spectrophotometer (Amersham Biosciences, NJ, USA). The recommended RNA quality parameters for the microarray analysis are an OD $260 / 280$ ratio in the range of $1.8-2.0$, an OD 260/230 ratio $>1.8$, a $18 \mathrm{~s} / 28 \mathrm{~s}$ rRNA ratio in the range of $1.8-2.1$, and an RNA integrity number (RIN) $>8.0$.

Microarray analysis of miRNA profiles. The human miRNA microarray ver. 2 kit (Agilent Technologies), which contains probes for 723 human and 76 human viral miRNAs was adapted to analyze the expression profiles of miRNAs. Before hybridizing the miRNA to the microarray, the total RNA (100 ng) including miRNAs was dephosphorylated with calf intestine alkaline phosphatase and denatured by adding DMSO and heating. The dephosphorylated RNA was labeled with pCp-Cy3 by T4 RNA ligase, and the labeled RNA was purified with a Micro Bio-Spin P-6 column (Bio-Rad Laboratories, CA, USA). The purified RNA was denatured and hybridized to the microarray at $55^{\circ} \mathrm{C}$ and $20 \mathrm{rpm}$ in an Agilent Microarray Hybridization Chamber (Agilent Technologies) for $20 \mathrm{~h}$. Then the microarray slide was washed with wash buffers and scanned with an Agilent scanner to obtain the microarray image. The numerical data for the miRNA profiles were extracted from the images with the Feature Extraction program (Agilent Technologies). These data were analyzed with GeneSpring GX software version 7.3 (Agilent Technologies).

qRT-PCR analysis of miRNAs. qRT-PCR was used to confirm the miRNA expression profiles obtained from the microarray data. The template miRNA cDNA was constructed from total RNA by reverse transcriptase with RT primers from miScript PCR Systems (Qiagen, Hilden, Germany). The PCR was conducted with a Line gene K Real-Time PCR machine (BIOER Technology Co., China). The SYBR and U6 gene were used for detecting the gene amplification and normalizing the each sample, respectively. Relative quantification of the miRNAs was calculated with
Pfaffl's model (37) after determining the PCR efficiency by the method of Rasmussen (38).

Target prediction of miRNAs. Candidate miRNAs, which changed more than 2-fold following radiation exposure, were picked for target prediction and analyzed with miRBase Targets Version 5 on the Enright Lab (Wellcome Trust Sanger Institute) website (http://microrna.sanger.ac.uk/targets/v5/). Human genes with experimentally verified functions related to apoptosis, cell cycle arrest, or DNA damage and repair, were selected from the Gene Ontology website (http://www. geneontology.org/). Finally, the genes predicted as targets by candidate miRNAs and those selected on the basis of gene ontology were aligned by their gene names, and genes appearing in both lists were chosen and listed.

\section{Results and Discussion}

We first wished to determine which miRNAs showed changes in expression in response to IR. To monitor the expression profiles of miRNAs following IR treatment, we employed a microarray containing probes to 723 human miRNAs. In our preliminary study, we found that A549 was less sensitive to radiation than other cell types (data not shown); therefore, we chose relatively high radiation doses (20 and $40 \mathrm{~Gy}$ ) for this study. At $24 \mathrm{~h}$ after IR exposure, total RNA was extracted from the cells and was used to probe the microarray. A miRNA was flagged as 'Present' if it yielded sufficiently high quality data in experimental conditions. In total, 202 miRNAs were flagged as Present, and their expression profiles following each radiation dose are shown in Fig. 1A. Although the expression levels for all miRNAs showed some variability, most miRNAs did not show strong response in expression following IR.

To identify the miRNAs with robust changes in expression following IR treatment, we carried out fold change analysis on the microarray data. In this analysis, 12 and 18 miRNAs in 20- and 40-Gy-treated cells, respectively, showed $>2$-fold changes compared to control cells (Fig. 1B). Interestingly, the fraction of up- versus down-regulated miRNAs was very different between the 20- and 40-Gy-treated cells. Among IR-responsive miRNAs, eight miRNAs were found to be changed following both the 20- and 40-Gy treatments. In contrast, four miRNAs were unique to the 20-Gy-treated cells and ten miRNAs showed expression changes only in the 40-Gy-treated cells (Fig. 1C). The IR-responsive miRNAs and their expression values are listed in Table I. Although we found that the fraction of strongly up- and down-regulated miRNAs differed between the two radiation doses, we noted that for each of the eight miRNAs showing expression changes following both doses of IR, the direction, but not necessarily the magnitude, of the expression change was the same for both radiation doses (Table I). However, the miRNAs such as miR-139-3p, $-34 b^{*},-22^{*}$ and $-34 a$ were included only either side of radiation, they were showing near the 2 -fold changes in the other radiation dose.

We next used qRT-PCR to confirm the expression profiles of five of the IR-responsive miRNAs identified in the microarray analysis. qRT-PCR analysis of miRNAs hsa-miR-16-2*, $-106 a,-139-3 p,-345$, and $-516 a-5 p$ is shown in Fig. 2. For each miRNA, the expression levels following 20- and 40-Gy 


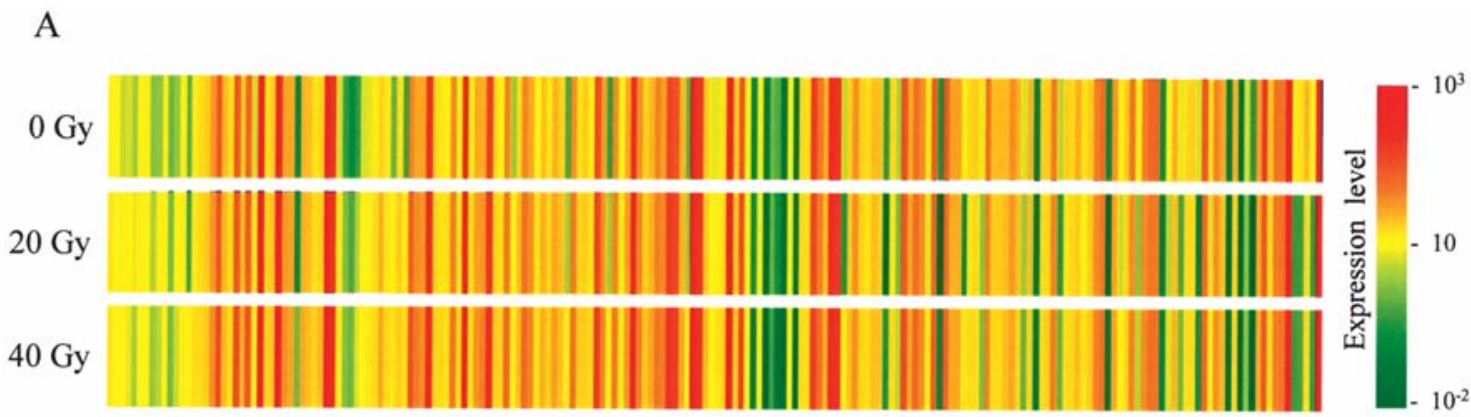

B

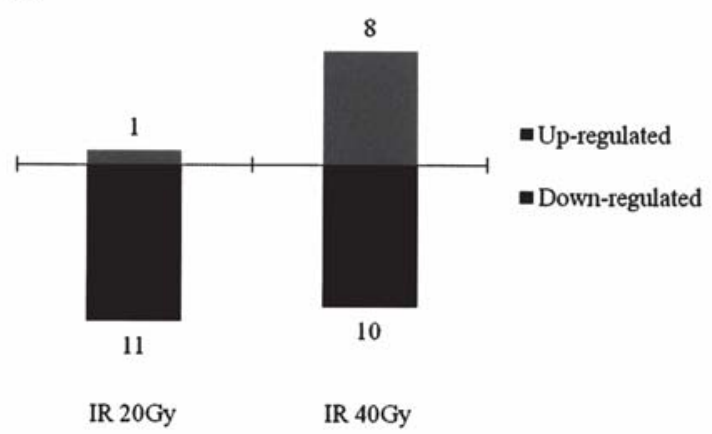

C

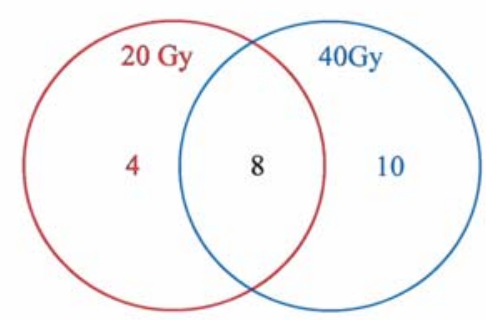

No changes : 180

Figure 1. miRNA profiles associated with the radiation doses. After microarray analysis, the expression profiles of miRNAs flagged as Present in the A549 cells were described by color ranges (A). Among the Present-flagged miRNAs, miRNAs that changed 2-fold were selected, and their regulated patterns are summarized according to the number of miRNAs up- or down-regulated by radiation (B). The miRNAs are presented in a Venn diagram depending on the radiation dosage $(\mathrm{C})$.

Table I. miRNAs changed more than 2-fold by at least one of the two radiation doses.

20 and 40 Gy

8

Only 40 Gy

10
Fold changes $^{\mathrm{a}}$

Inclusion

No. of miRNAs

miRNA name

20 Gy

40 Gy

Only 20 Gy

4

hsa-miR-636

hsa-miR-593

hsa-miR-760

$-25.2$

$-1.1$

hsa-miR-139-3p

$-13.5$

$-2.9$

$-1.4$

hsa-miR-345

$-2.6$

$-1.2$

hsa-miR-885-3p

$-39.1$

$-1.9$

hsa-miR-206

$-8.1$

$-58.3$

hsa-miR-516a-5p

$-3.9$

$-9.5$

hsa-miR-16-2*

$-3.2$

$-2.0$

hsa-miR-106a

$-3.0$

$-2.6$

$-2.2$

hsa-miR-548c-3p

$-2.2$

$-2.2$

$-25.4$

hsa-miR-127-3p

hsa-miR-1228*

hsa-miR-30b*

hsa-miR-376a

hsa-miR-34b*

hsa-miR-215

hsa-miR-183

hsa-miR-22*

hsa-miR-34a

hsa-miR-192

hsa-miR-30c- ${ }^{*}$
2.7

$-5.2$

$-3.1$

2.0

$-1.2$

$-1.3$

2.0

1.4

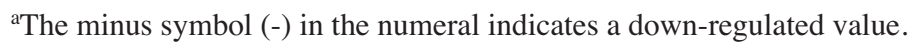


A

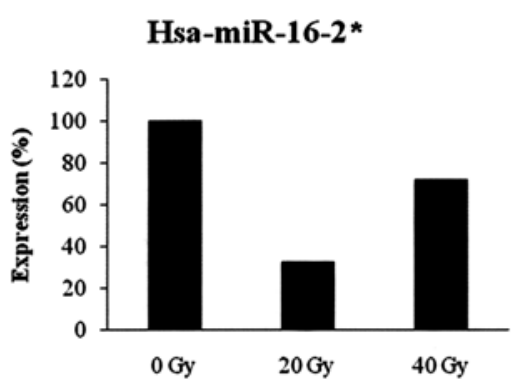

D

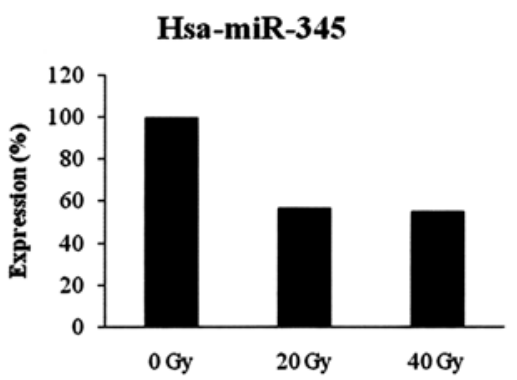

B

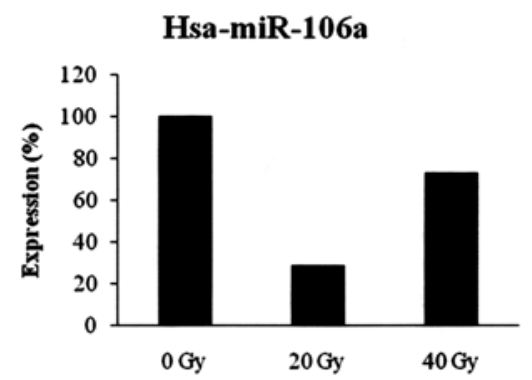

$\mathrm{E}$

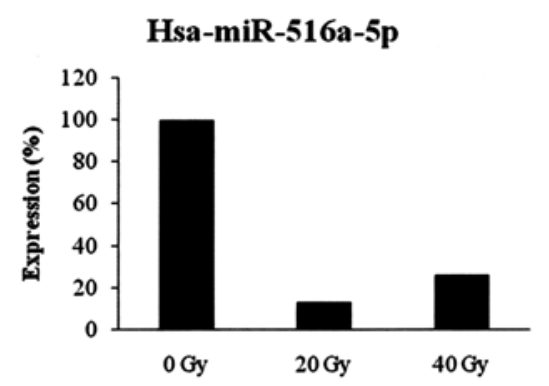

$\mathrm{C}$

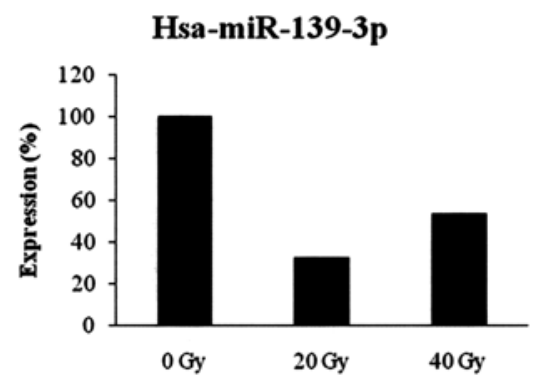

Figure 2. Quantification and confirmation of miRNA expression levels by qRT-PCR. Among the miRNAs that changed more than 2-fold, five miRNAs, hsa-miR-16-2* (A), hsa-miR-106a (B), hsa-miR-139-3p (C), hsa-miR-345 (D), and hsa-miR-516a-5p (E), were selected, and their expression levels were confirmed by qRT-PCR. The expression levels were compared with those in 0 Gy-irradiated cells (controls normalized as 100).

IR treatments were compared to those of control cells, the value of which was set to 100 . For all five miRNAs, the qRT-PCR experiment confirmed their down-regulation by IR. However, the magnitude of the changes in the expression levels was found to be different between the microarray and qRT-PCR analyses. In particular, miR-345 showed the largest discrepancy between the microarray and qRT-PCR data. Whereas the microarray data indicated a 50-fold decrease in mir-345 expression after IR, the qRT-PCR analysis revealed only a 2 -fold change.

To begin to identify potential targets of the IR-responsive miRNAs identified in the fold-change analysis, we conducted a bioinformatical analysis directed at identifying potential targets having functions related to apoptosis, cell cycle control, and DNA damage and repair. We chose genes with these functions, since these represent the main responses observed in IR-treated cells. First, the targets of the IR-responsive miRNAs were predicted with a miRNA target prediction tool, miRBase Targets Version 5. For each miRNA, approximately 1,000 potential target genes were predicted (data not shown). However, no data was available in the target prediction tool for miR-1228*. Next, we used the Gene Ontology website to produce lists of genes having experimentally confirmed functions related to apoptosis, cell cycle regulation, or DNA damage and repair. These lists contained 172, 76 and 82 genes, respectively. Finally, genes from miRNA target prediction were compared with those from the gene ontology analysis, and the overlaps between these sets of genes are listed in Table II. We found that more of the predicted targets had functions related to apoptosis compared to cell cycle control and DNA repair. Some of the genes from this analysis were predicted to be targets of more than one miRNA. As reported by John et al (39), one miRNA has the potential to target many mRNAs, and a single mRNA could be regulated by several miRNAs.

Among the IR-responsive miRNAs with potential roles in apoptosis and cell cycle arrest that we have identified, miR-34a is the best characterized (40-45): it is directly transactivated by p53 and is involved in the regulation of apoptosis, cell cycle arrest, and senescence-like growth arrest; it also functions by targeting many genes, including SIRT1, CCND1, CDK6, and by modulating the activity of pathways such as androgen receptor (AR)-dependent p53-induced apoptosis and the E2F pathway. Although these genes were not among those predicted by miRBase, another prediction program, TargetScan 4.2, predicted them as targets of miR-34a (data not shown). This may indicate that target predictions vary greatly between different programs, and that researchers must carefully analyze potential targets. In addition, miR-34a also has tumor suppressor activity through the targeting of oncogenes such as MYCN, and aberrant $\mathrm{CpG}$ methylation or deletion of $1 \mathrm{p} 36$, the chromosomal location of miR-34a, is observed in many types of cancer (46-48).

In this study, we have demonstrated that a subset of human miRNAs show robust expression changes in response to IR. Given the broad use of radiation in cancer therapy and biological studies, it is important to note that miRNAs may have unidentified roles in the cellular response irradiation. Although we have not proved that the potential miRNA targets are directly regulated by the miRNAs identified in this study, we have highlighted the possibility of miRNAmediated regulation of the radiation response. Future studies will be required to identify the precise mechanisms underlying this regulation. 
Table II. Prediction of apoptosis-, cell cycle control-, or DNA damage and repair-related targets of miRNAs from Table I.

\begin{tabular}{|c|c|c|c|}
\hline \multirow[b]{2}{*}{ miRNA name } & \multicolumn{3}{|c|}{ Functions of target genes } \\
\hline & Apoptosis & Cell cycle and arrest & DNA damage and repair \\
\hline hsa-miR-16-2* & CADM1, GTF2H3, HSPA5, NRG1 & BTG3, KNTC1 & $\begin{array}{l}\text { EXO1, MSH2, PCNA, } \\
\text { POLD4, SETX }\end{array}$ \\
\hline hsa-miR-22* & $\begin{array}{l}\text { AIFM3, CASP8, CIDEC, IL6, RTKN, } \\
\text { TP53BP2, TRADD }\end{array}$ & ZW10 & EXO1, HMGB1, WRN \\
\hline hsa-miR-30c- $1^{*}$ & $\begin{array}{l}\text { CARD8, CASP8, DFFB, ERCC3, } \\
\text { TNFRSF10B, TRADD }\end{array}$ & CCND3, GMNN & POLD4, POLL, ERCC3 \\
\hline hsa-miR-30b* & $\begin{array}{l}\text { AGT, BCL3, DDB2, ERCC3, PML, } \\
\text { RTKN, TP53 }\end{array}$ & PML & $\begin{array}{l}\text { ERCC1, ERCC3, GTF2H4, } \\
\text { PML, POLD4, RPA1, TP53 }\end{array}$ \\
\hline hsa-miR-34a & $\begin{array}{l}\text { APOH, BAX, BID, BNIPL, DEDD2, } \\
\text { FAS, GRIK2, PCBP4, RYR2, TGFB1 }\end{array}$ & $\begin{array}{l}\text { CD28, DBC1, PCBP4, } \\
\text { TCF3, TGFB1 }\end{array}$ & PCBP4, POLD1 \\
\hline hsa-miR-34b* & BAX, BNIPL, BRE, FAS, NLRP2, SCG2 & ATR, DBC1, SPHK1, TCF3 & ATR, ERCC5, RFC5 \\
\hline hsa-miR-106a & $\begin{array}{l}\text { BAX, BNIPL, HBXIP, NUP62, P2RX4, } \\
\text { PPP3R1, TNFRSF10B }\end{array}$ & BTG3 & GTF2H3, MSH3, POLH \\
\hline hsa-miR-127-3p & AIFM3, BAD, BCL6, NLRP1, PPP3CC & CCND3, RBM38 & NEIL1, RBM38, RPA1 \\
\hline hsa-miR-139-3p & $\begin{array}{l}\text { ADAMTSL4, AKT1, BAD, BRE, } \\
\text { CASP10, CDC2, DNAJB6, MAEA, } \\
\text { NLRP1 }\end{array}$ & BRSK1, GTPBP4, TCF3 & POLA1, POLE \\
\hline hsa-miR-183 & $\begin{array}{l}\text { CASP10, DFFB, GRIK2, HIP1, IL12B, } \\
\text { LCK, PPP3CC, SRA1, TCF7L2 }\end{array}$ & $\begin{array}{l}\text { BTG3, CD28, CDKN3, } \\
\text { HEXIM2, IL12B, NUSAP1 }\end{array}$ & EXO1 \\
\hline hsa-miR-192 & $\begin{array}{l}\text { ALB, ANGPTL4, ERCC } 3 \text {, FURIN, } \\
\text { TCF7L2 }\end{array}$ & SPHK1 & $\begin{array}{l}\text { CDK7, ERCC3, ERCC4, } \\
\text { XPA }\end{array}$ \\
\hline hsa-miR-206 & $\begin{array}{l}\text { CIDEC, HSPD1, RASA1, SCG2, TBX3, } \\
\text { TCF7L2 }\end{array}$ & CDKN3, SPHK1, TBX3 & - \\
\hline hsa-miR-215 & ALB, ERCC3, FURIN, P2RX4, TCF7L2 & SPHK 1 & $\begin{array}{l}\text { CDK7, ERCC3, ERCC4, } \\
\text { TDG, XPA }\end{array}$ \\
\hline hsa-miR-345 & ANGPTL4, DHCR24, KNTC1, PCBP4 & ZW10 & $\begin{array}{l}\text { LIG1, POLD3, POLL, } \\
\text { RFC1, RPA1 }\end{array}$ \\
\hline hsa-miR-376a & $\begin{array}{l}\text { CADM1, CASP8, FAS, HBXIP, IL6, } \\
\text { PPP3CC, TERF1 }\end{array}$ & $\begin{array}{l}\text { ATR, BTG3, CDT1, } \\
\text { GMNN, NEK11 }\end{array}$ & ATR, MNAT1, NEK11 \\
\hline hsa-miR-516a-5p & $\begin{array}{l}\text { ADAMTSL4, BRCA1, CASP8, CD27, } \\
\text { CIDEB, FURIN, MAEA, TNF }\end{array}$ & BMP4 & $\begin{array}{l}\text { ASF1A, BRCA1, GTF2H4, } \\
\text { MBD4 }\end{array}$ \\
\hline hsa-miR-548c-3p & AGT, CD70, DFFB, FAS & AFAP1L2, EGF, GMNN & $\begin{array}{l}\text { CDK7, GTF2H1, HMGB2, } \\
\text { MBD4, MPG, MSH2, } \\
\text { PCNA, PMS2 }\end{array}$ \\
\hline hsa-miR-593 & CD27, DDB2, DYNLL1, RYR2, STK4 & - & - \\
\hline hsa-miR-636 & AGT, BFAR, COL4A3, P2RX4, TRAF2 & DBC1 & ERCC4, ERCC5, OGG1 \\
\hline hsa-miR-760 & $\begin{array}{l}\text { ACIN1, BRCA1, BRE, CASP10, CIDEC, } \\
\text { HTATIP2, IL6, LGALS12 }\end{array}$ & CDT1, SPHK1 & BRCA1 \\
\hline hsa-miR-885-3p & $\begin{array}{l}\text { AIFM3, AKT1, BNIP1, CDKN2A, } \\
\text { CDKN2C, INHA, SFN, TCF7L2 }\end{array}$ & $\begin{array}{l}\text { CDKN2A, CDKN2C, } \\
\text { EDN3, ZW10 }\end{array}$ & $\begin{array}{l}\text { DDB1, POLD1, PRKCG, } \\
\text { RFC5 }\end{array}$ \\
\hline hsa-miR-1228* & N/A & N/A & N/A \\
\hline
\end{tabular}




\section{Acknowledgements}

This work was supported by the Ministry of Education, Science andTechnology (2007-03151) and the Ministry of Knowledge Economy (R-2006-1-043) of the Republic of Korea.

\section{References}

1. Lee RC, Feinbaum RL and Ambros V: The C.elegans heterochronic gene lin-4 encodes small RNAs with antisense complementarity to lin-14. Cell 75: 843-854, 1993.

2. Lagos-Quintana M, Rauhut $\mathrm{R}$, Lendeckel $\mathrm{W}$ and Tuschl T: Identification of novel genes coding for small expressed RNAs. Science 294: 853-858, 2001.

3. Lau NC, Lim LP, Weinstein EG and Bartel DP: An abundant class of tiny RNAs with probable regulatory roles in Caenorhabditis elegans. Science 294: 858-862, 2001

4. Lee RC and Ambros V: An extensive class of small RNAs in Caenorhabditis elegans. Science 294: 862-864, 2001.

5. Bernstein E, Caudy AA, Hammond SM and Hannon GJ: Role for a bidentate ribonuclease in the initiation step of RNA interference. Nature 409: 363-366, 2001.

6. Lai EC: MicroRNAs are complementary to 3'-UTR motifs that mediate negative post-transcriptional regulation. Nat Genet 3: 363-364, 2002.

7. Gregory RI, Chendrimada TP, Cooch N and Shiekhattar R: Human RISC couples microRNA biogenesis and posttranscriptional gene silencing. Cell 123: 631-640, 2005.

8. Ørom UA, Nielsen FC and Lund AH: MicroRNA-10a binds the 5'UTR of ribosomal protein mRNAs and enhances their translation. Mol Cell 30: 460-471, 2008.

9. Place RF, Li LC, Pookot D, Noonan EJ and Dahiya R: MicroRNA-373 induces expression of genes with complementary promoter sequences. Proc Natl Acad Sci USA 105: 1608-1613, 2008.

10. Lee Y, Jeon K, Lee JT, Kim S and Kim VN: MicroRNA maturation: stepwise processing and subcellular localization. EMBO J 21: 4663-4670, 2002.

11. Lee Y, Kim M, Han J, et al: MicroRNA genes are transcribed by RNA polymerase II. EMBO J 23: 4051-4060, 2004

12. Denli AM, Tops BB, Plasterk RH, Ketting RF and Hannon GJ: Processing of primary microRNAs by the microprocessor complex. Nature 432: 231-235, 2004.

13. Hammond SM, Bernstein E, Beach D and Hannon GJ: An RNA-directed nuclease mediates post-transcriptional gene silencing in Drosophila cells. Nature 404: 293-296, 2000.

14. Ketting RF, Fischer SE, Bernstein E, et al: Dicer functions in RNA interference and in synthesis of small RNA involved in developmental timing in C.elegans. Genes Dev 15: 2654-2659, 2001.

15. Yi R, Qin Y, Macara IG and Cullen BR: Exportin-5 mediates the nuclear export of pre-microRNAs and short hairpin RNAs. Genes Dev 17: 3011-3016, 2003.

16. Alvarez-Garcia I and Miska EA: MicroRNA functions in animal development and human disease. Development 132: 4653-4662, 2005.

17. Kent OA and Mendell JT: A small piece in the cancer puzzle: microRNAs as tumor suppressors and oncogenes. Oncogene 2 6188-6196, 2006.

18. Zhang B, Pan X, Cobb GP and Anderson TA: MicroRNAs as oncogenes and tumor suppressors. Dev Biol 302: 1-12, 2007.

19. Lin SL, Chang DC, Chang-Lin S, et al: Mir-302 reprograms human skin cancer cells into a pluripotent ES-cell-like state. RNA 14: 2115-2124, 2008.

20. Marson A, Levine SS, Cole MF, et al: Connecting microRNA genes to the core transcriptional regulatory circuitry of embryonic stem cells. Cell 134: 521-533, 2008.

21. Berezikov E, Guryev V, van de Belt J, et al: Phylogenetic shadowing and computational identification of human microRNA genes. Cell 120: 21-24, 2005

22. Lewis BP, Burge CB and Bartel DP: Conserved seed pairing, often flanked by adenosines, indicates that thousands of human genes are microRNA targets. Cell 120: 15-20, 2005.

23. Baek D, Villén J, Shin C, et al: The impact of microRNAs on protein output. Nature 455: 64-71, 2008.

24. Selbach M, Schwanhäusser B, Thierfelder N, et al: Widespread changes in protein synthesis induced by microRNAs. Nature 455: 58-63, 2008.
25. Rosenfeld N, Aharonov R, Meiri E, et al: MicroRNAs accurately identify cancer tissue origin. Nat Biotechnol 26: 462-469, 2008.

26. Schmidt-Ullrich RK, Dent P, Grant S, Mikkelsen RB and Valeie K: Signal transduction and cellular radiation responses. Radiat Res 153: 245-257, 2000.

27. Barlow C, Brown KD, Deng CX, Tagle DA and WynshawBoris A: Atm selectively regulates distinct p53-dependent cellcycle checkpoint and apoptotic pathways. Nat Genet 17: 453-456, 1997.

28. Canman CE, Lim DS, Cimprich KA, et al: Activation of the ATM kinase by ionizing radiation and phosphorylation of $\mathrm{p} 53$. Science 281: 1677-1679, 1998.

29. Iliakis G, Wang Y, Guan J and Wang H: DNA damage checkpoint control in cells exposed to ionizing radiation. Oncogene 22: 5834-5847, 2003.

30. Cann KL and Hicks GG: Regulation of the cellular DNA double-strand break response. Biochem Cell Biol 85: 663-674, 2007.

31. Schwartz JL: The radiosensitivity of the chromosomes of the cells of human squamous cell carcinoma cell lines. Radiat Res 129: 96-101, 1992.

32. Schwartz JL, Murnane J and Weichselbaum RR: The contribution of DNA ploidy to radiation sensitivity in human tumour cell lines. Br J Cancer 79: 744-747, 1999.

33. Cuneo KC, Fu A, Osusky K, Huamani J, Hallahan DE and Geng L: Histone deacetylase inhibitor NVP-LAQ824 sensitizes human nonsmall cell lung cancer to the cytotoxic effects of ionizing radiation. Anticancer Drugs 18: 793-800, 2007.

34. Kasten-Pisula U, Windhorst S, Dahm-Daphi J, Mayr G and Dikomey E: Radiosensitization of tumour cell lines by the polyphenol Gossypol results from depressed double-strand break repair and not from enhanced apoptosis. Radiother Oncol 83: 296-303, 2007.

35. Brognard J, Clark AS, Ni Y and Dennis PA: Akt/protein kinase B is constitutively active in non-small cell lung cancer cells and promotes cellular survival and resistance to chemotherapy and radiation. Cancer Res 61: 3986-3997, 2001.

36. Toulany M, Kehlbach R, Florczak U, et al: Targeting of AKT1 enhances radiation toxicity of human tumor cells by inhibiting DNA-PKcs-dependent DNA double-strand break repair. Mol Cancer Ther 7: 1772-1781, 2008.

37. Pfaffle MW: A new mathematical model for relative quantification in real-time RT-PCR. Nucleic Acids Res 29: e45, 2001.

38. Rasmussen R: Quantification on the light cycler. In: Rapid Cycle Real-time PCR: Methods and Applications. Meuer S, Wittwer C and Nakagawara K (eds.) Springer Press, Heidelberg, pp21-34, 2001.

39. John B, Enright AJ, Aravin A, et al: Human microRNA targets. PLoS Biol 2: e363, 2004.

40. Chang TC, Wentzel EA, Kent OA, et al: Transactivation of miR-34a by p53 broadly influences gene expression and promotes apoptosis. Mol Cell 26: 745-752, 2007.

41. Tarasov V, Jung P, Verdoodt B, et al: Differential regulation of microRNAs by $\mathrm{p} 53$ revealed by massively parallel sequencing: miR-34a is a p53 target that induces apoptosis and G1-arrest. Cell Cycle 6: 1586-1593, 2007.

42. Rokhlin W, Scheinker VS, Taghiyev AF, et al: MicroRNA-34 mediates AR-dependent p53-induced apoptosis in prostate cancer. Cancer Biol Ther 7: 1288-1296, 2008.

43. Sun F, Fu H, Liu Q, et al: Downregulation of CCND1 and CDK6 by miR-34a induces cell cycle arrest. FEBS Lett 582 : 1564-1568, 2008.

44. Tazawa H, Tsuchiya N, Izumiya $M$ and Nakagama H: Tumorsuppressive miR-34a induces senescence-like growth arrest through modulation of the E2F pathway in human colon cancer cells. Proc Natl Acad Sci USA 104: 15472-15477, 2008.

45. Yamakuchi M, Ferlito M and Lowenstein CJ: miR-34a repression of SIRT1 regulates apoptosis. Proc Natl Acad Sci USA 105: 13421-13426, 2008.

46. Cole KA, Attiyeh EF, Mosse YP, et al: A functional screen identifies miR-34a as a candidate neuroblastoma tumor suppressor gene. Mol Cancer Res 6: 735-742, 2008.

47. Lodygin D, Tarasov V, Epanchintsev A, et al: Inactivation of miR-34a by aberrant CpG methylation in multiple types of cancer. Cell Cycle 7: 2591-2600, 2008.

48. Wei JS, Song YK, Durinck S, et al: The MYCN oncogene is a direct target of miR-34a. Oncogene 27: 5204-5213, 2008. 